\title{
A simple, clinically relevant therapeutic vaccine shows long-term protection in an aggressive, delayed-treatment B lymphoma model
}

\author{
Pallab Pradhan, Jardin Leleux, Jiaying Liu, and Krishnendu Roy \\ Wallace H. Coulter Department of Biomedical Engineering at Georgia Tech and Emory, Center for Immunoengineering \\ at Georgia Tech, Parker H. Petit Institute for Bioengineering and Biosciences, Georgia Institute of Technology, \\ Atlanta, Georgia, USA.
}

\begin{abstract}
Despite initial remission after successful treatments, B lymphoma patients often encounter relapses and resistance causing high mortality. Thus, there is a need to develop therapies that prevent relapse by providing long-term protection and, ultimately, lead to functional cure. In this study, our goal was to develop a simple, clinically relevant, and easily translatable therapeutic vaccine that provides durable immune protection against aggressive $B$ cell lymphoma and identify critical immune biomarkers that are predictive of long-term survival. In a delayed-treatment, aggressive, murine model of A20 B lymphoma that mimics human diffuse large B cell lymphoma, we show that therapeutic A2O lysate vaccine adjuvanted with an NKT cell agonist, $\alpha$-galactosylceramide ( $\alpha$-GalCer), provides long-term immune protection against lethal tumor challenges and the antitumor immunity is primarily CD8 T cell dependent. Using experimental and computational methods, we demonstrate that the initial strength of germinal center reaction and the magnitude of class-switching into a Th1 type humoral response are the best predictors for the long-term immunity of B lymphoma lysate vaccine. Our results not only provide fundamentally insights for successful immunotherapy and long-term protection against B lymphomas, but also present a simple, therapeutic vaccine that can be translated easily due to the facile and inexpensive method of preparation.
\end{abstract}

Conflict of interest: The authors have declared that no conflict of interest exists.

Submitted: December 27, 2016

Accepted: October 17, 2017

Published: November 16, 2017

Reference information: JCI Insight. 2017;2(22):e92522. https://doi.org/10.1172/jici. insight.92522.

\section{Introduction}

The American Cancer Society estimates that in 2017 about 72,240 people in the United States will be diagnosed with non-Hodgkin lymphomas (NHL), more than half of these people will be in advanced stages, and approximately 20,140 patients will die of the disease. About $85 \%$ of NHL cases are B cell lymphomas, and among them majority are diffuse large B cell lymphoma (DLBCL). Even with the advent of better diagnosis and new treatments, such as rituximab (a monoclonal anti-CD20 antibody that has significantly increased 5-year survival rates $[1,2])$, B cell lymphomas remain a major burden to health and society and a deadly disease for all age groups. Effective therapeutic vaccines and immunotherapy approaches, which can be readily translated into humans and can significantly enhance current clinical practice to offer long-term functional "cure," could be transformative.

Tumor antigen-specific therapeutic vaccines allow tumor-specific immune responses to be generated and reduce the chance of autoimmune cross-reactivity of the resulting $\mathrm{T}$ cells. However, this approach requires that a specific tumor antigen is known and that the antigen is either highly overexpressed or exclusively expressed (the latter being the preferred option) on tumor cells. For B lymphomas, a well-established tumorspecific antigen (idiotype [Id]) has been used as tumor vaccine in numerous preclinical settings as well as in clinical trials. Due to its poor immunogenicity, the Id antigen is often used as Id-keyhole limpet hemocyanin conjugate (Id-KLH) (3-6). Significant progress has been made over the last decade, and currently, patientspecific Id antigen-based therapeutic vaccines are in clinical trials $(3,4,7,8)$. A recently completed, randomized, double-blinded, multicenter phase III trial showed an increase $(P=0.045)$ in disease-free survival of follicular lymphoma patients treated with an Id-KLH+GM-CSF vaccine over KLH+GM-CSF alone; but this treatment strategy failed to enhance overall survival of Id-KLH antigen treatment groups over controls (4). 
A A20 tumor challenge ( $1 \times 10^{5}$ cells/mice, IP)
A20 tumor lysate vaccination

Day

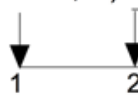

A20 tumor rechallenge ( $2 \times 10^{5}$ cells/mice, IP)
B

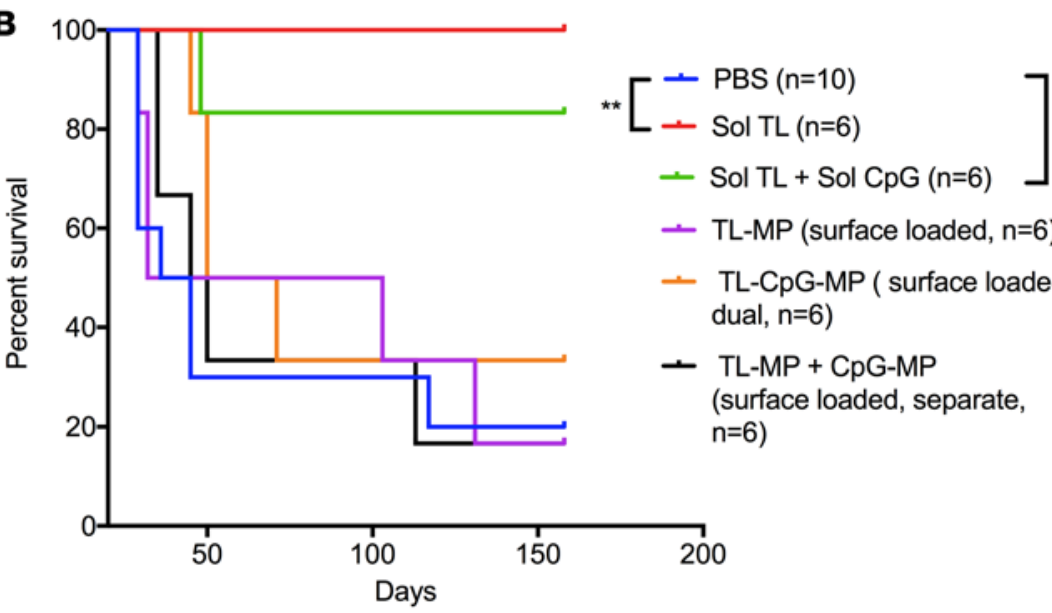

Figure 1. Therapeutic efficacy of various A20 lysate vaccine formulations. (A) Time line for therapeutic immunization and tumor rechallenge studies in 1-day preestablished A20 B cell lymphoma model. (B) Kaplan-Meier curve ( $n=6-10$ per group) showing survival for A20 tumor lysate formulation-vaccinated mice (at $100 \mu$ g lysate protein/mouse, s.c.) after 2 lethal A20 tumor challenges (dose, $1 \times 10^{5}$ cells/mouse, i.p., on day 1 , rechallenged with $2 \times 10^{5}$ cells/mouse, i.p., on day 80$)$ in a therapeutic immunization setting. ${ }^{*} P$ $<0.05$, ${ }^{* *} P<0.01$, log-rank (Mantel-Cox) test. (C) Time line for therapeutic immunization in 7-day preestablished A20 B cell lymphoma model. (D) Kaplan-Meier survival curve for various $A 20$ tumor lysate formulation-injected BALB/C mice ( $n=10$ per group) following lethal $A 20$ tumor challenges with $2 \times 10^{5}$ cells, i.p.

\section{C}
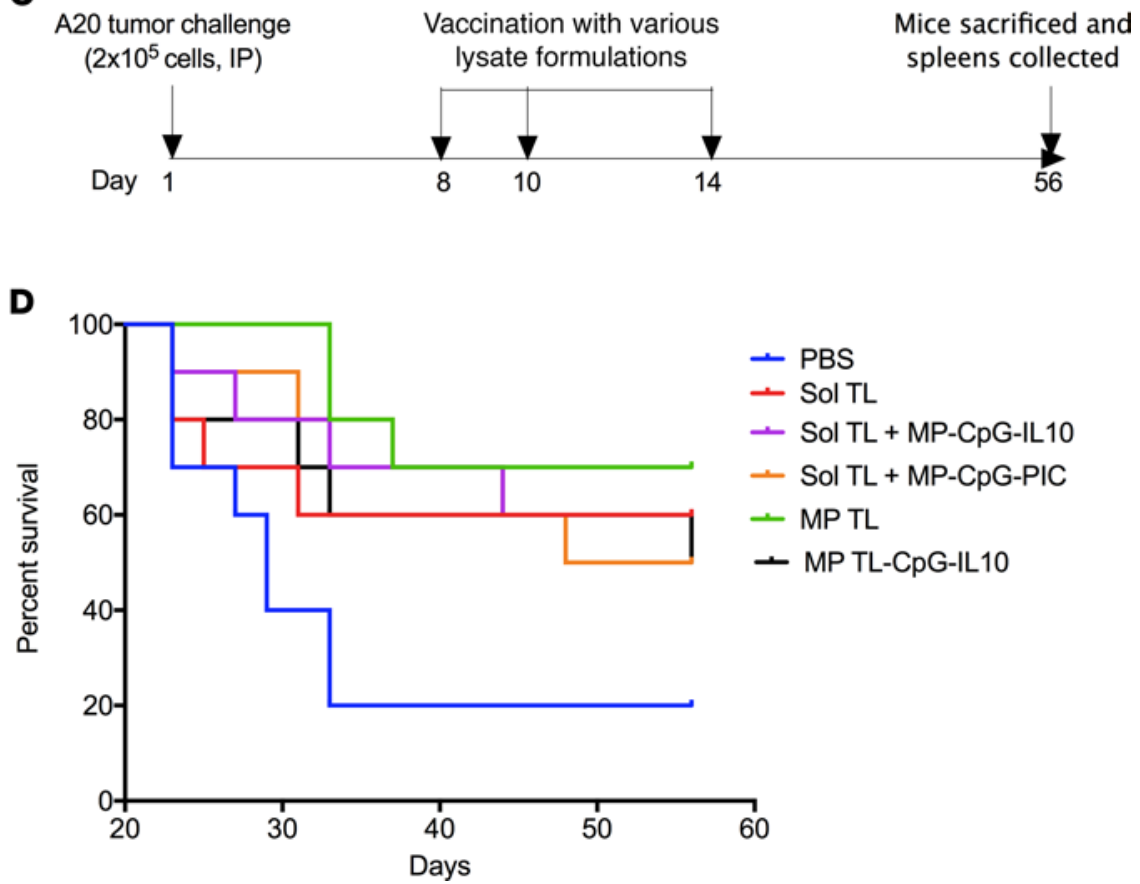

Since the Id antigen is different for every patient, preparation of personalized Id protein vaccine using recombinant hybridoma technology is very expensive and time consuming (9). Although, Id antigen-specific DNA vaccine reduces production time and cost, and is easier to scale compared with protein vaccine, therapeutic efficacy of Id DNA vaccine for B cell lymphoma has been modest at best, even when used with various molecular and biomaterial-based adjuvants $(10,11)$. In fact, these DNA vaccines have only showed good protective response in the A20 B cell lymphoma model (a well-established murine model of DLBCL, ref. 12) when coinjected with either highly toxic myotoxin (13) or with concurrent, long-term daily dosing of lenalidomide (14), both of which would be difficult to translate in humans. Hence, there remains an urgent need to develop a therapeutic B cell lymphoma vaccine that is highly effective, provides long-term protection in therapeutic models that are more indicative of the late- 
Table 1. Median survival and log-rank $P$ values and hazard ratios (compared with the PBS group) for Figure 1D

\begin{tabular}{lccc}
\hline Groups & P value (log-rank c/w PBS) & Median survival (d) & Hazard ratio (log-rank, c/w PBS) and 95\% Cl of ratio \\
PBS $(n=10)$ & - & 29 & - \\
Sol TL $(n=10)$ & 0.129 & $>56$ & $0.4307(0.1385-1.34)$ \\
Sol TL + MP-CpG-IL10 siRNA $(n=10)$ & 0.0527 & $>56$ & $0.3516(0.1114-1.109)$ \\
Sol TL + MP-CpG-PIC $(n=10)$ & 0.0566 & 52 & $0.3761(0.1224-1.156)$ \\
MP TL $(n=10)$ & $0.0063^{A}$ & $>56$ & $0.2147(0.0628-0.7331)$ \\
MP TL-CpG-IL10 siRNA $(n=10)$ & 0.1245 & 56 & $0.4581(0.1524-1.377)$ \\
AP $<0.01$ log-rank (Mantel-Cox) test. c/w PBS, compared with PBS. & & \\
\hline
\end{tabular}

stage human disease (i.e., high tumor burden, delayed treatment models), and could be relative simple to translate into clinic. In addition, little is known about what immune biomarkers are most predictive of long-term therapeutic protection in advanced B lymphomas. Thus, studies that identify such potential biomarkers are important for clinically relevant therapies.

Tumor lysate-based vaccine allows a patients' own immune system to "choose" the antigens by presenting the complete protein-peptide repertoire of the tumor to antigen-presenting cells (e.g., DCs). It also ensures that DCs are presented with other "not currently known" antigens, even if a known tumor antigen exists for that particular tumor. This approach also allows for a quick, low-cost way of immunizing patients, since the cells can be obtained from biopsy or blood draw, minimally manipulated and formulated (lysed), and injected back with appropriate adjuvants. However, this comes with a cost, an increased probability of autoimmune cross-reactivity: i.e., the chance that in the presence of potent adjuvants, DCs can get sensitized to other, nontumorigenic self-proteins and cause autoimmune reactions. Nevertheless, lysatebased vaccines are being investigated as a viable immunotherapy approach for a variety of tumors (15-18), and in several clinical trials no significant autoimmune reactions were observed $(15,19)$. For lymphoma, there are few reports on tumor lysate-pulsed DC vaccines (20-22). However, directly administered therapeutic tumor lysate vaccines against $B$ lymphomas have not been reported.

In this study, our goal was to (a) develop an effective tumor lysate-based therapeutic vaccine for B cell lymphoma that ensures long-term protection against tumor rechallenge in an aggressive, high tumor burden mouse model of DLBCL and (b) identify the immune biomarkers that are most correlative with longterm protection in DLBCL. We found that therapeutic A20 tumor lysate vaccine, when combined with an NKT cell agonist, $\alpha$-galactosylceramide ( $\alpha$-GalCer), provides durable immune protection against lethal tumor challenge in an aggressive therapeutic model of A20 B cell lymphoma. We also found, using both experimental and computational methods, that the strength of germinal center reaction and class-switching into a Th1-type humoral response is most predictive of long-term protection in this model of DLBCL.

\section{Results}

Therapeutic lysate vaccine provides long-term immune protection against lethal A20 B cell lymphoma challenges in mice. We first tested the therapeutic efficacy of various tumor lysate formulations - soluble lysate (Sol TL) with or without the TLR9 ligand CpG (Sol CpG) or tumor lysate surface-loaded on to biodegradable polymer microparticles (MP-surface TL) with or without CpG (separate/dual loaded with lysate on MPs) - in a 1-day preestablished A20 B cell lymphoma model in mice. This is not an aggressive, high-tumor burden model, but it represents one where very early diagnosis can be made and the tumor burden, although ultimately lethal, is moderate. In this study, mice were first challenged with a lethal dose of A20 cells $\left(1 \times 10^{5} \mathrm{~A} 20\right.$ cells, i.p. $)$ and then immunized with soluble tumor lysate (100 $\mu$ g lysate protein, s.c.) at days 2,7 , and 15 (Figure 1A). Further, a rechallenge (representing relapse) with double the lethal dose of A20 tumor cells $\left(2 \times 10^{5}\right.$ A20 cells, i.p.) was done in surviving mice at day 80 to access long-term immune protection (Figure 1A). Interestingly, A20 tumor lysate alone protected $100 \%$ mice against two lethal challenges, whereas only $20 \%$ mice survived in the PBS group up to day 160 (Figure 1B). Combined treatment of Sol CpG and Sol TL showed similar therapeutic effect as Sol TL alone, indicating no survival benefit of CpG. However, none of the tumor lysate surface loaded on microparticle (MP) groups had any survival benefit over controls. These results indicate that therapeutic A20 tumor lysate vaccine, even without any adjuvant, could provide long-term immune protection against lethal challenge in a therapeutic model of early-stage B cell lymphoma. 
Encouraged by excellent long-term immune protection of the TL vaccine in the early-stage model, we tested its therapeutic efficacy in a more aggressive, high tumor burden $\left(2 \times 10^{5}\right.$ cells, i.p. injected $)$, delayed treatment (7-day preestablished) DLBCL model. In addition to Sol TL, we tested different particulate adjuvant formulations to further enhance therapeutic efficacy of A20 tumor lysate vaccine. One of the formulations was a slow-release formulation for A20 TL, in which we encapsulated the lysate in MPs (MP TL; average size, $1.8 \mu \mathrm{m}$ ) with high encapsulation efficiency (about $75 \%$ at $5 \%$ weight ratio of lysate protein to polymer) (Supplemental Figure 1; supplemental material available online with this article; https://doi. org/10.1172/jci.insight.92522DS1). MP TL showed a slow and sustained release profile, releasing about $34 \%$ lysate protein over a 6 -week period in vitro (Supplemental Figure 1). Further, we modified the surface of MP TL with a polycation, PEI, using EDC/NHS chemistry to enable surface coloading of a TLR9 agonist, CpG, and IL10-silencing siRNA (described as MP TL-CpG-IL10 group) as we reported previously $(10,23)$. We also synthesized MPs without encapsulated lysate to prepare two other particulate adjuvant formulations, MP surface coloaded with CpG and IL10 siRNA (referred to as MP-CpG-IL10) and CpG and Poly (I:C) (coded as MP-CpG-PIC) by electrostatic interaction as we reported recently (10). In this study, mice were immunized 3 times with various formulations 7 days after high-dose A20 tumor inoculation ( $2 \times 10^{5}$ A20 cells, i.p.) (Figure 1C). Here, prolonged survival of A20 tumor-challenged mice was found in all the formulation-treated groups (Figure 1D). Moreover, all the lysate vaccine groups, including the Sol TL group, exhibited an extended median survival of mice to over 52 days, from 29 days in the PBS group (Table 1). Notably, the MP TL group a showed significant increase in survival of mice. However, particulate adjuvant groups, MP-CpG-IL10 siRNA or MP-CpG-PIC, did not have significant effect on enhancing immune protection when combined with A20 tumor Sol TL. Further analysis of splenocytes of mice sacrificed on day 56 revealed that the Sol TL group had a significantly higher percentage of IFN- $\gamma$ secreting CD8 T cells and NK cells (Supplemental Figure 2, A and B). On the other hand, the MP TL group showed a significantly higher percentage of NK cells, without any increase in IFN- $\gamma$-secreting CD8 $\mathrm{T}$ cell response compared with naive mice. Moreover, all the tested particulate adjuvant groups showed a significantly higher percentage of NK cells compared with naive mice. Notably, the Sol TL and MP TLCpG-IL10 groups had a significantly higher percentage of myeloid-derived suppressor cells (MDSCs) than naive mice (Supplemental Figure 2C). Treg levels were similar across the groups (Supplemental Figure 2D).

Therapeutic soluble A20 tumor lysate vaccine adjuvanted with NKT cell agonist, $\alpha$-GalCer, provides long-term, durable immune protection against lethal A20 tumor challenges in mice. Next we studied whether the long-term protective efficacy soluble tumor lysate (Sol TL) and its particle-encapsulated formulation (MP TL) can be further enhanced using non-TLR vaccine adjuvants. Given our earlier observation that NK cells and IFN-secreting T cells could be involved in protection and that $\mathrm{CpG}$ or poly (I:C) did not increase survival, we chose an NKT cell-stimulatory agent, $\alpha$-GalCer, as an adjuvant, which is known to indirectly increase NK and T cell responses due to the bystander effect of NKT cell stimulation $(24,25)$. Recently, $\alpha$-GalCerloaded autologous irradiated whole tumor cells were shown to enhance antitumor immunity in the murine E $\mu$-myc lymphoma model but only showed short-term protection (about 40 days) (25).

Long-term, durable immune protection is highly desirable for a therapeutic vaccine to prevent relapse of cancer, a very common problem of many cancers, including B cell lymphoma. To test long-term immune protection of various tumor lysate vaccines (e.g., Sol TL and MP TL with/without $\alpha$-GalCer), we first injected mice with a lethal dose A20 tumor cells $\left(2 \times 10^{5}\right.$ cells, i.p. $)$ and then immunized mice with Sol TL or MP TL at days 8, 10, and 14 (Figure 2A). On day 17, a single injection of $\alpha$-GalCer $(2 \mu \mathrm{g}$, i.p.) was given to selected groups and a second challenge of a lethal dose of A20 tumor $\left(2 \times 10^{5}\right.$ cells, i.p.) was performed on day 56 (Figure 2A). Mouse survival was tracked long term (up to 128 days). Immunization with only soluble tumor lysate (Sol TL) showed significantly higher survival (with a median survival of 56 days) as compared with PBS-treated groups (median survival, 33 days) (Figure 2B and Table 2). Strikingly, single injection of $\alpha$-GalCer following Sol TL immunizations prolonged survival of mice further, with a median survival of 100 days. However, MP-encapsulated tumor lysate (MP TL, median survival, 46.5 days) performed relatively worse than the Sol TL group (median survival, 56 days) in providing immune protection to lethally challenged mice (Figure $2 B$ and Table 2). In contrast to the Sol TL plus $\alpha$-GalCer group, the MP TL plus $\alpha$-GalCer group did not show higher median survival of mice over the MP TL-treated group (Table 2). Taken together, these results suggest that therapeutic soluble A20 tumor lysate vaccine when combined with the NKT cell agonist, $\alpha$-GalCer, provides long-term, durable immune protection in an aggressive, delayed-treatment A20 B lymphoma model.

$\alpha$-GalCer treatment enhances $C T L, N K T$, and NK cell responses of therapeutic soluble A20 lysate vaccine. To mechanistically understand the immune responses of A20 lysate vaccines and identify potential bio- 
A

A20 tumor challenge ( $2 \times 10^{5}$ cells, IP)
A20 lysate vaccination

\section{a-GalCer inj}

A20 tumor rechallenge

$\left(2 \times 10^{5}\right.$ cells, IP)
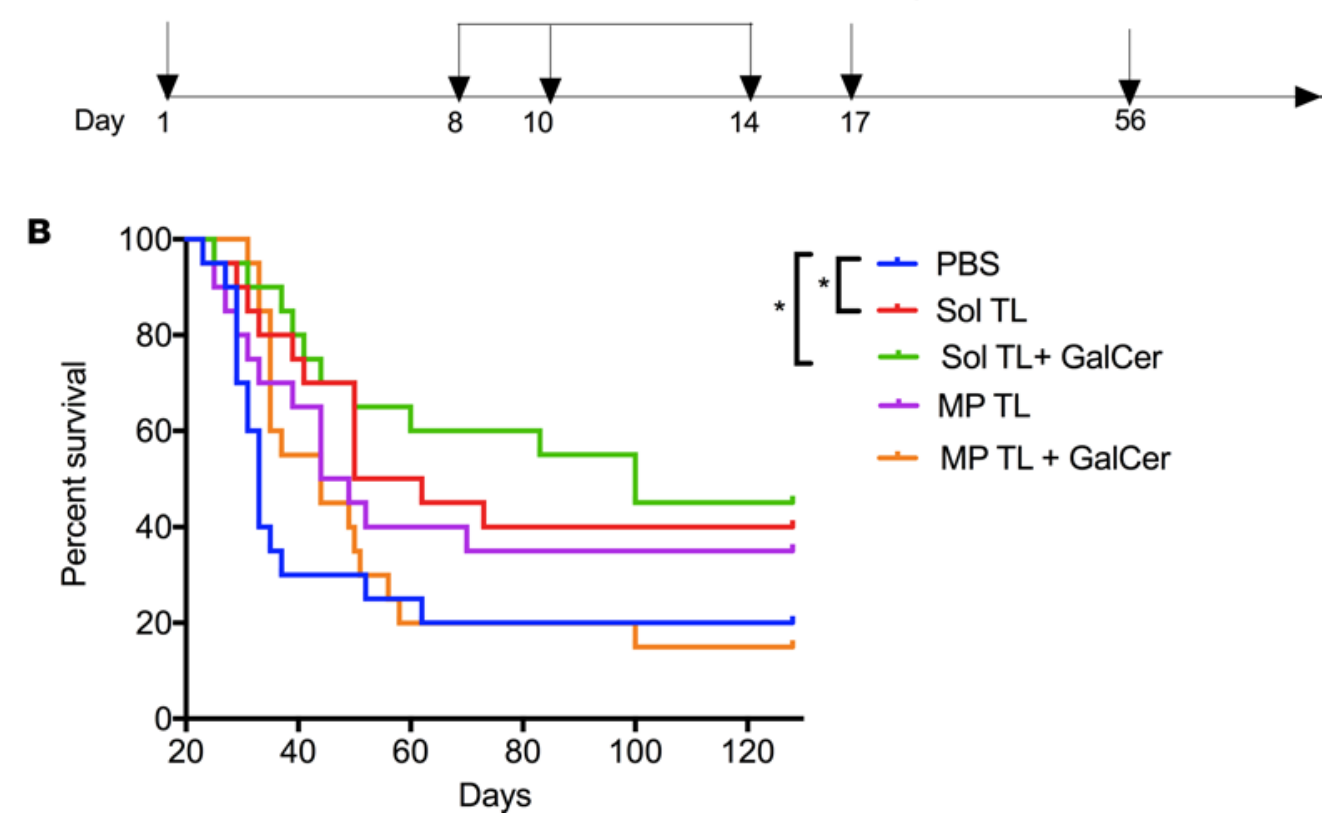

Figure 2. Therapeutic efficacy of soluble and microparticle-encapsulated A20 lysate vaccine formulations in 7-day preestablished A20 B-lymphoma model. (A) Time line for therapeutic immunization and tumor rechallenge studies in 7-day preestablished A20 B cell lymphoma model. (B) Kaplan-Meier survival curve ( $n=20$ per group, pooled data from 2 independent experiments) for various A20 tumor lysate formulation-treated mice after 2 lethal A20 tumor challenges of $2 \times 10^{5}$ cells/mouse. Naive BALB/C mice were first injected (i.p.) with a lethal dose of A20 cells ( $2 \times 10^{5}$ cells/mouse), followed by 3 immunizations at days 8,10 , and 14 with various tumor lysate formulations (100 $\mu$ g lysate protein/mouse, s.c.) and at day 17 with $\alpha$-GalCer ( $2 \mu \mathrm{g} / \mathrm{mouse}$, i.p.). A second challenge with $2 \times 10^{5}$ A20 tumor cells/mouse, i.p., was done at day 56 , and moue survival was followed up to 128 days. ${ }^{*} P<0.05,1$-way ANOVA with Tukey multiple comparison tests.

markers for survival, we sacrificed 5-6 mice from each therapeutic group at day 21 (timeline shown in Figure 3A) and analyzed cellular and humoral immunity using serum, spleen, and inguinal lymph nodes. All the lysate vaccine treatment groups showed a higher CD4 T cell-mediated cytotoxic $\mathrm{T}$ cell (CTL) response compared with the PBS group (Figure 3A). Notably, the Sol TL plus $\alpha$-GalCer group outperformed other groups with highest percentage of CD4-CTL response. Furthermore, the Sol TL plus $\alpha$-GalCer group was the only group that showed a higher CD8-CTL response than the PBS group (Figure $3 \mathrm{~B})$. On the other hand, as expected, $\alpha-G a l C e r$ treatment enhanced the NKT cell response for both the Sol TL and MP TL vaccine groups (Figure 3C and Supplemental Figure 3A). Moreover, the Sol TL and $\alpha$-GalCer combination showed a significantly higher NK cell response over the Sol TL group, but none of the vaccine treatment groups had significantly higher percentage of NK cells compared with the PBS group (Figure 3D and Supplemental Figure 3B). Collectively, these results indicate that $\alpha$-GalCer treatment enhances CTL and NKT responses for the Sol TL vaccine.

$\alpha$-GalCer treatment enhances lysate antigen-specific IFN- $\gamma$ and IL4 responses of therapeutic soluble A20 lysate vaccine. Lysate antigen-specific $\mathrm{T}$ cell response and secreted cytokine level were assessed by splenocyte restimulation study. Here, the Sol TL group showed higher Th1 (IFN- $\gamma$, TNF- $\alpha$, and IL2) and Th2 (IL4, IL10, and IL5) cytokine levels in the culture media compared with the PBS group (Figure 3, $\mathrm{E}$ and $\mathrm{F}$, and Supplemental Figure 4). Interestingly, $\alpha-G a 1 C e r$ treatment preferably increased IFN- $\gamma$ and IL4 levels in the restimulation media (Figure 3, E and F). Both the Sol TL and Sol TL plus $\alpha$-GalCer groups had higher percentages of IFN- $\gamma$-secreting $\mathrm{CD} 8^{+}$and $\mathrm{CD} 4^{+} \mathrm{T}$ cells as compared with the PBS group (Supplemental Figure 5). However, the MP TL and MP TL plus $\alpha$-GalCer groups showed only minimal levels of Th1 or Th2 cytokine in restimulation media (Figure 3, E and F, and Supplemental Figure 4). Overall, these results suggest that combined treatment of Sol TL and $\alpha$-GalCer enhances lysate antigen-specific IFN- $\gamma$ (Th1) and IL4 (Th2) responses.

$\alpha$-GalCer treatment with soluble A20 lysate vaccine decreases both granulocytic MDSCS and Tregs in splenocytes. Immune regulatory cells, MDSCs, and Tregs play critical roles in dampening the antitumor immune 
Table 2. Median survival and log-rank $P$ values and hazard ratios (compared with the PBS group) for Figure 2B

\begin{tabular}{lccc}
\hline Groups & P value (log-rank c/w PBS) & Median survival (d) & Hazard ratio (log-rank, c/w PBS) and 95\% Cl of ratio \\
PBS $(n=20)$ & - & 33 & - \\
Sol TL $(n=20)$ & $0.0434^{A}$ & 56 & $0.4896(0.2295-1.045)$ \\
Sol TL + GalCer $(n=20)$ & $0.0121^{\mathrm{A}}$ & 100 & $0.4021(0.184-0.8787)$ \\
MP TL $(n=20)$ & 0.1719 & 46.5 & $0.6183(0.2966-1.289)$ \\
MP TL + GalCer $(n=20)$ & 0.3705 & 44 & -
\end{tabular}

${ }^{A} P<0.05$, log-rank (Mantel-Cox) test. c/w PBS, compared with PBS.

response. We analyzed MDSC and Treg populations in splenocytes following therapeutic immunization with various A20 tumor lysate vaccine formulations. We observed moderate to high level of MDSCs (both granulocytic and monocytic) and Tregs in the PBS groups at day 21 after lethal challenge with A20 tumor cells (Figure 3, G and H). Sol TL vaccine did not alter MDSCs but decreased Tregs in splenocytes as compared with the PBS group (Figure 3, G and H, and Supplemental Figure 6). However, $\alpha$-GalCer treatment with Sol TL vaccine markedly decreased both granulocytic MDSCs and Tregs in splenocytes (Figure 3, $\mathrm{G}$ and H, and Supplemental Figure 6). Notably, the MP TL plus $\alpha$-GalCer group showed higher levels of granulocytic MDSCs and Tregs than the Sol TL plus $\alpha$-GalCer group (Figure 3, G and H, and Supplemental Figure 6). Collectively, the Sol TL plus $\alpha$-GalCer treatment group effectively decreased both granulocytic MDSCs and Tregs in spleens of A20 tumor-challenged mice.

$\alpha$-GalCer treatment enhances germinal center and humoral responses of therapeutic soluble A20 lysate vaccine. The germinal center is known to play a critical role in shaping vaccine humoral response. We assessed germinal center response for lysate vaccines in lymph nodes by immunohistochemistry and flow cytometry. The Sol TL plus $\alpha$-GalCer group showed the highest number of germinal centers per draining lymph node section (Figure 4A). Moreover, $\alpha$-GalCer treatment significantly increased percentages of both germinal center-forming B cells and total B cells for Sol TL vaccine (Figure 4, B and C, and Supplemental Figure 7A); however, these responses were low for both MP TL and MP TL plus $\alpha$-GalCer groups. We also measured anti-A20 tumor lysate antibody level in serum using ELISA and detected a high level of anti-A20 tumor lysate IgG1 antibody (at day 21) across all vaccine groups (Figure 4D). Interestingly, $\alpha$-GalCer treatment enhanced IgG1 antibody level even further for the Sol TL vaccine group. However, we did not see a similar trend in IgG2a antibody level (at day 21) and the PBS control group showed high OD (Figure 4E). This is due to the fact that A20 tumor cells are IgG2a-type B lymphoma cells (show high surface IgG2a expression, Supplemental Figure 7B) and they contain high amounts of IgG2a in their lysates, resulting in high background signal in A20 lysate-coated ELISA assays.

Next, we assessed long-term humoral response by measuring serum anti-A20 tumor lysate antibody level at day 128 for the mice that survived 2 lethal tumor challenges (on days 1 and 56) in the therapeutic immunization studies (Figure 2A). We observed high levels for both IgG1 and IgG2a across all vaccine groups (Figure 4, F and $G)$. Interestingly, $\alpha$-GalCer treatment moderately increased antitumor lysate IgG2a antibody levels for Sol TL vaccine, indicating a Th1-biased humoral response (Figure 4G). We also found that the serum antibody, specifically IgG1, triggered by lysate vaccines specifically binds to A20 B lymphoma cells; however, we did not see a similar trend in specific binding for serum IgG2a antibody, which could be due to nonspecific surface staining of highly expressed surface IgG2a on A20 cells (Supplemental Figure 7C). Further, we measured humoral responses of lysate vaccine against known A20 B lymphoma-specific Id antigen. Lysate vaccines, specifically Sol TL and Sol TL plus $\alpha$-GalCer, triggered strong humoral responses against Id antigen with high serum levels of anti-A20 Id-IgG1 antibody (Supplemental Figure 7D). Notably, the titer of anti-lysate antibody was higher than the titer of anti-A20 antibodies, indicating that the lysate vaccine generates antibodies against other antigens in addition to the known tumor antigens.

Murine cell lines are often known to be contaminated with murine leukemia virus (MuLV). To determine if any viral contamination plays a role in generating potent antitumor immunity, we first tested our A20 cells (purchased from ATCC) to detect MuLV and found that this cell line is positive for MuLV-related proteins in ELISA (0.3 ng MuLV Gag-P30 protein/ $\mu g$ A20 cell lysate) and MuLV transcripts in qPCR assays (MuLV $C_{T}, 12.06$; $\beta$-actin $\left.C_{T}, 16.26\right)$. Interestingly, to our knowledge this is the first report that demonstrates MuLV contamination of A20 lymphoma cells. Next, to rule out any specific role of MuLV antigens in the observed immune response of A20 lysate 
A A20 tumor challenge ( $2 \times 10^{5}$ cells, IP)

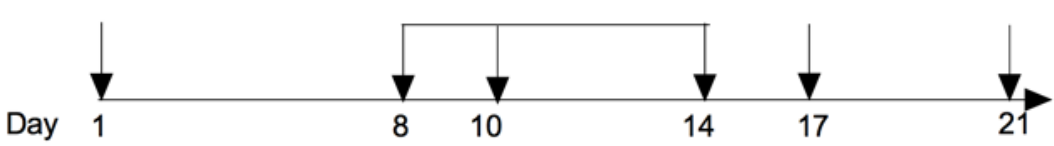

A20 lysate

vaccination
B
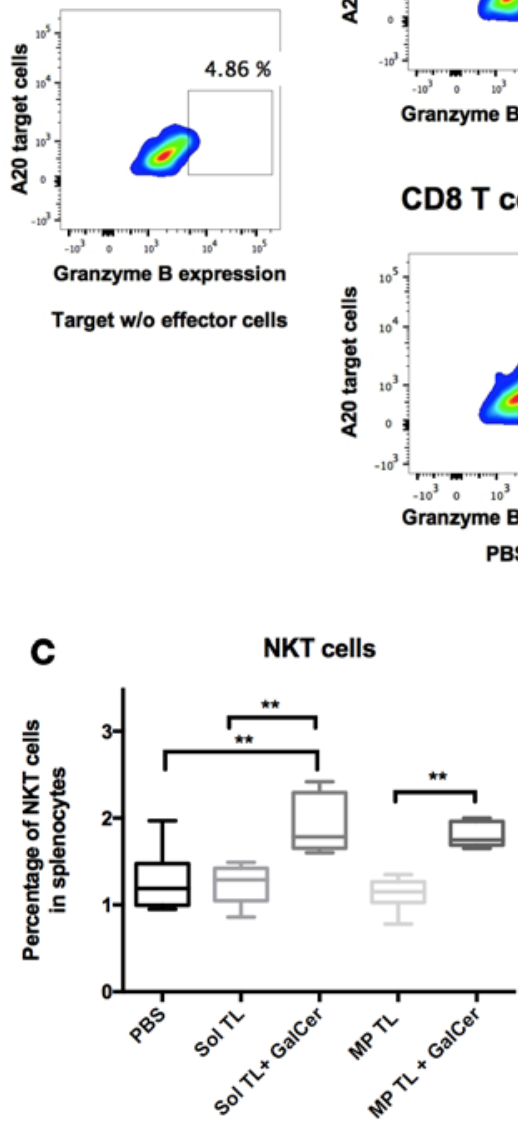

F

IL4
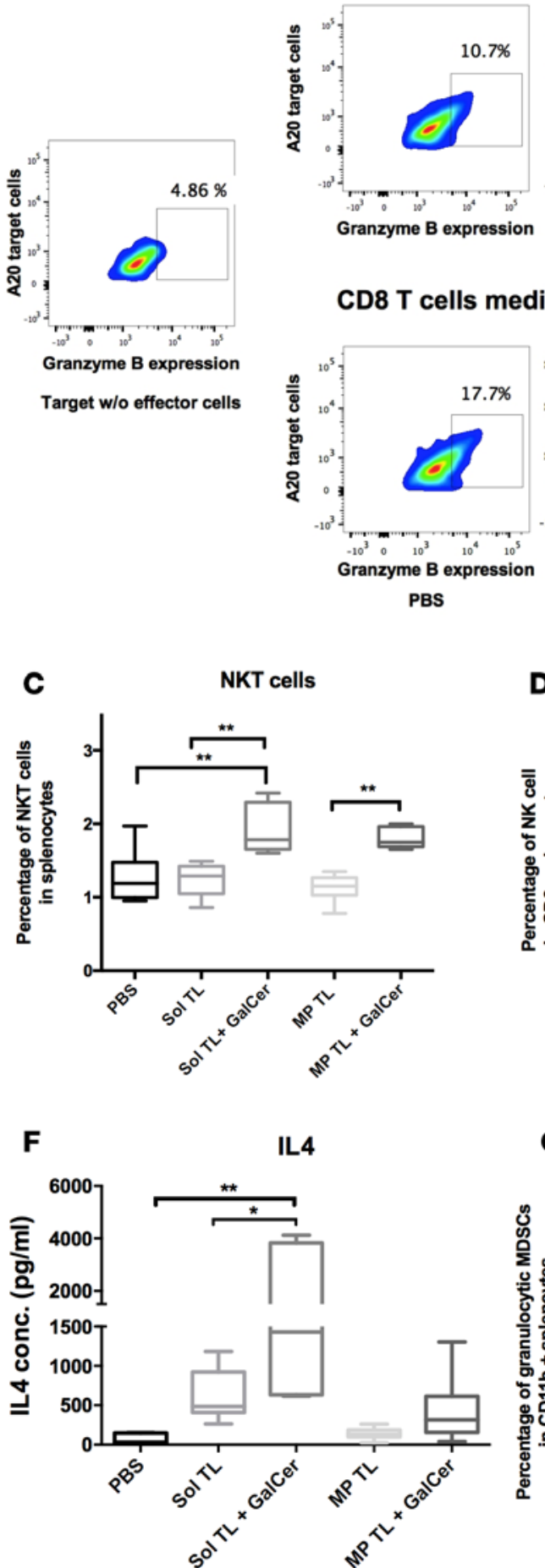

a-GalCer inj Mechanistic studies

CD4 T cells mediated CTL response
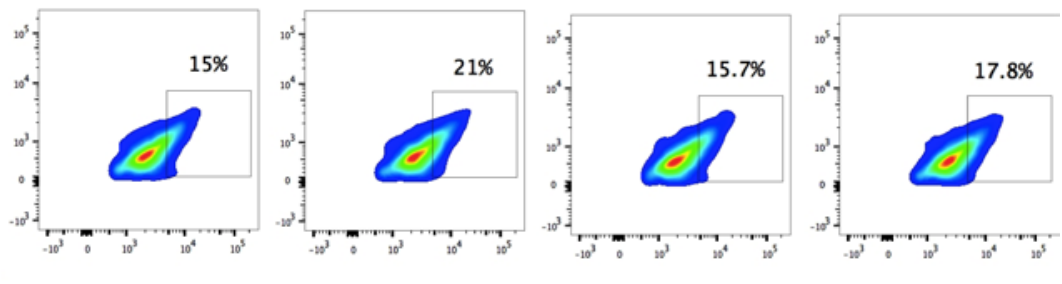

\section{CD8 T cells mediated CTL response}
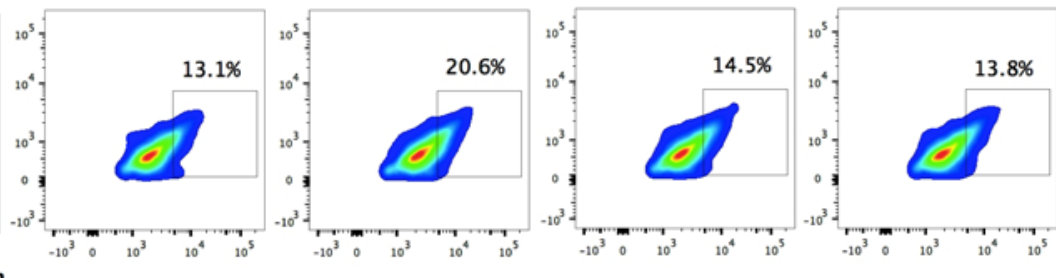

Sol TL

Sol TL + GalCer

MP TL

MP TL + GalCer

D

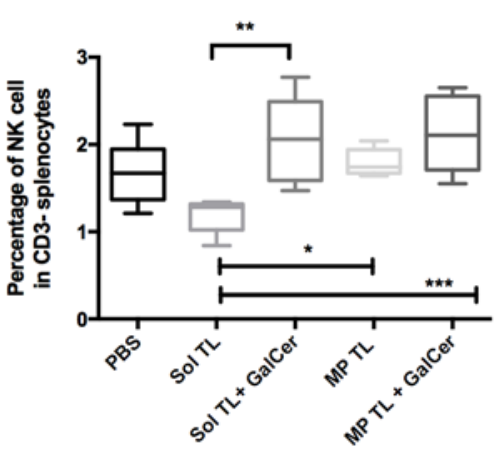

G

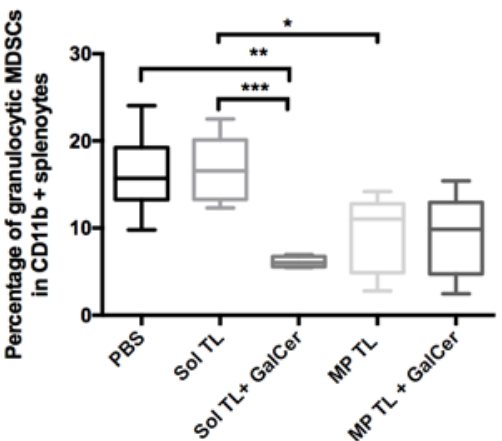

E

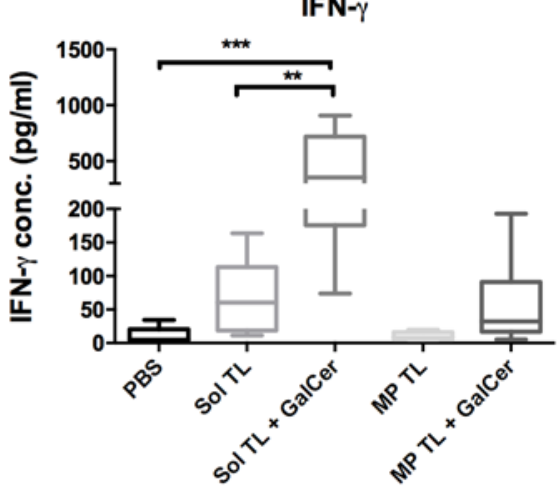

H

Tregs (CD4+CD25+FOXP3+)

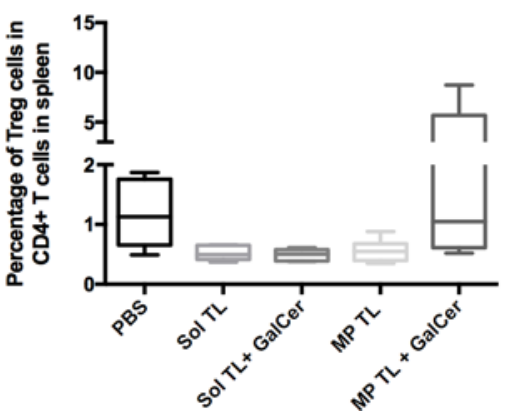

Figure 3. Cellular immune responses for various therapeutic A20 lysate vaccine formulations. (A) Time line for mechanistic studies. BALB/C mice ( $n$ $=5-6$ ) were first injected with $2 \times 10^{5}$ A20 tumor cells on day 1 and then immunized with the formulations on day 8, 10, and 14. Thereafter, a single i.p. injection of $2 \mu \mathrm{g} \alpha$-GalCer was done at day 17 only in $\alpha$-GalCer treatment groups. On day 21, all the mice were sacrificed and spleens were collected and 
analyzed for CTL, NKT, and NK cell responses by flow cytometry. (B) CD4 ${ }^{+}$and CD8 ${ }^{+}$T cell-mediated CTL response by granzyme B assay. Effector CD4+ and $\mathrm{CD8}^{+}$T cells were magnetically sorted from pooled splenocytes for each group. (C) Box plot showing NKT cell percentage in splenocytes $(n=6)$. (D) Box plot showing NK cell percentage in splenocytes $(n=6)$. (E and F) IFN- $\gamma$ (Th1 cytokine) and IL4 (Th2 cytokine) secreted by splenocytes following ex vivo restimulation with A20 lysate antigens $(n=6)$. Splenocytes $\left(10^{6} /\right.$ well of 96-well plate) were restimulated with A20 lysate (100 $\mu$ g/well) for 72 hours in vitro, and the culture media were analyzed for various Th1/Th2 cytokines using a multiplex luminex kit. (G) Box plot showing percentage of granulocytic MDSCs in CD11b splenocytes $(n=6)$. (H) Treg percentage in spleen CD4+ T cells $(n=5-6)$. Data represent mean \pm SD. ${ }^{*} P<0.05,{ }^{* *} P<0.01,{ }^{* * *} P<0.001$, 1-way ANOVA with Tukey multiple comparison tests.

vaccine, we measured anti-MuLV antigen-specific antibodies (moloney MuLV capsid protein 330 and glycosylated Gag poly protein) in the serum of tumor-challenged surviving mice at day 128. The anti-MuLV-p30 IgG1 antibody level was higher in the PBS and MP TL plus $\alpha$-GalCer groups (Supplemental Figure 7E); however, these groups were the worst performing groups in terms of median survival following two A20 tumor challenges. Moreover, the anti-MuLV-p30 IgG1 level was minimal (background levels) in the best performing group, Sol TL plus $\alpha$-GalCer. Anti-MuLV-gag polyprotein IgG1 was very low for all the groups except the MP TL group. Together, these results suggest that the MuLV antigen-specific humoral response did not have any significant role in enhancing median survival for A20 lysate vaccines.

CD8 T cells are the primary effector T cells for antitumor immunity of therapeutic soluble A20 lysate plus $\alpha$ GalCer vaccine. Next, we performed CD4 and CD8 T cell depletion studies to define the effector T cells that play a critical role in antitumor immunity for therapeutic soluble A20 lysate plus $\alpha$-GalCer vaccine (time line shown in Figure 5A). Further, to find whether NKT cells have any direct effect on tumor protection, we used anti-CD1d antibody to block CD1d- $\alpha$-GalCer-mediated NKT cell activation. Depletion of CD8 $\mathrm{T}$ cells significantly reduced survival for Sol TL plus $\alpha$-GalCer-treated mice, whereas CD4 T cell depletion did not show a significant effect on survival (Figure 5B). In addition, anti-CD1d antibody-treated mice did not show any change in survival for Sol TL plus $\alpha$-GalCer vaccine, indicating that the NKT cells do not play a direct role in antitumor protection. Taken together, these results suggest that CD8 T cells are the primary effector cells for antitumor immunity for therapeutic soluble A20 lysate plus $\alpha$-GalCer vaccine.

Linear discriminant analysis and principal component analysis identified that germinal center and Th1type humoral responses are potential predictive biomarkers for long-term immune protection of therapeutic soluble A20 lysate and $\alpha$-GalCer vaccine. Mechanistic results, as shown in Figures 3 and 4, indicate that multiple arms of innate and adaptive immune systems were activated by various therapeutic tumor lysate vaccine formulations. To get a better resolution of vaccine-specific immune response profile, we performed linear discriminant analysis (LDA) using JMP 12 software. Figure 6 shows canonical plots for cellular and humoral immune profiles of various lysate vaccine groups. From these plots, it is clearly evident that $\alpha$-GalCer treatment resulted into strong NKT and NK cell responses for both Sol TL and MP TL groups (Figure 6A). On the other hand, the Sol TL group exclusively had a TNF- $\alpha$ response and the Sol TL plus $\alpha$-GalCer group predominantly had a IFN- $\gamma$ response, but both MP TL and MP TL plus $\alpha$-GalCer groups failed to show theses cytokine responses upon restimulation of splenocytes with tumor lysate antigen ex vivo (Figure 6B). Although humoral responses of all the lysate vaccine groups were much stronger compared with the PBS group, the germinal center-forming B cell response was stronger in Sol TL and Sol TL plus $\alpha$-GalCer groups, whereas antitumor lysate IgG1 response was dominant in MP TL and MP TL plus $\alpha$-GalCer groups (Figure 6C). However, none of the vaccine groups showed remarkable discrimination in Tregs and granulocytic MDSCs responses (Figure 6D). We could not analyze CD4-CTL and CD8-CTL data using LDA, as both CD4-CTL and CD8-CTL responses were determined from pooled splenocytes for each group. Concurrently, we analyzed mechanistic and median survival data (mean for each immune response) using principal component analysis (PCA) to identify possible correlations of various immune parameters with median survival. Median survival was comparatively more correlated with CD4 CTL and NKT cell responses compared with CD8 CTL and NK cell responses, but it was very strongly correlated with IFN- $\gamma$ and IL4 cytokine responses (Supplemental Figure 8, A-C). However, correlation between median survival and IFN- $\gamma$-producing CD4 or CD8 T cells was not strong (Supplemental Figure 8D). Tregs and granulocytic MDSCs were negatively correlated with median survival (Supplemental Figure $8 \mathrm{E})$. In addition, it is interesting to note that the median survival correlated well with $\mathrm{B}_{2} 20^{+} \mathrm{GL} 7^{+}$germinal center-forming B cell response but correlated poorly with IgG1-d21 level (i.e., antitumor lysate IgG1 antibody level at day 21) (Supplemental Figure 8F). However, median survival showed a higher correlation with antitumor lysate IgG2a level (correlation coefficient, 0.6223) than IgG1 level (correlation coefficient 0.4606 ) at day 128 (Supplemental Figure 8G). Collectively, LDA and PCA analysis suggest that strong germinal center response (germinal center number and $\mathrm{B} 220^{+} \mathrm{BL} 7^{+}$cells) and Th1 response (IFN- $\gamma$ and IgG2a 
A
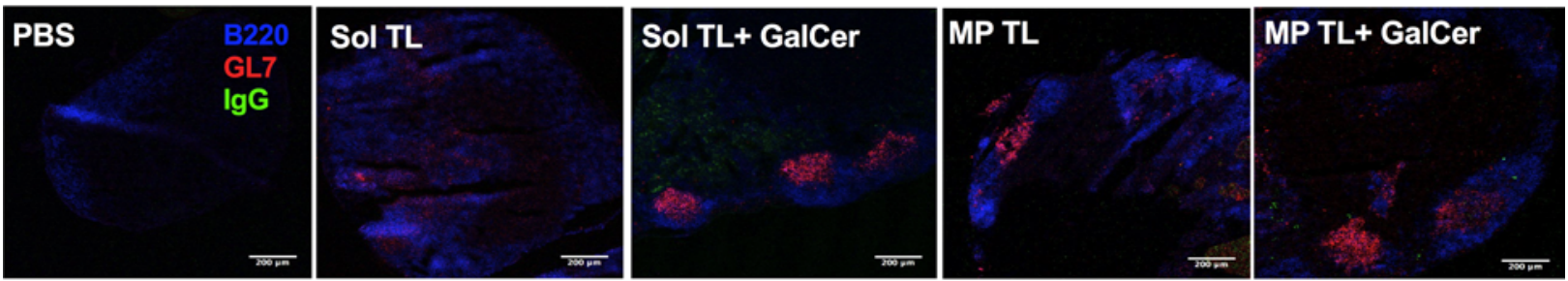

B B220+GL7+ B cells (lymph

C $\begin{gathered}\text { B cells } \\ \text { (lymph node) }\end{gathered}$

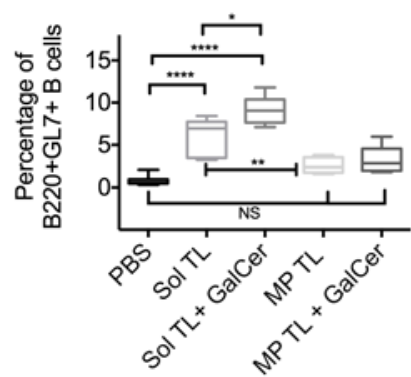

E

Anti lysate-lgG2a at day 21

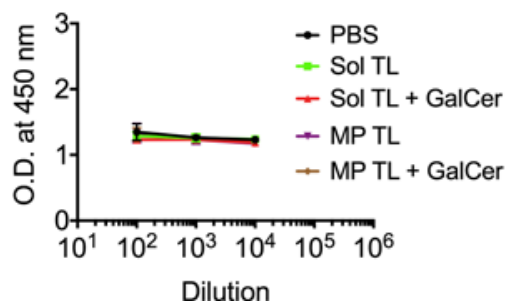

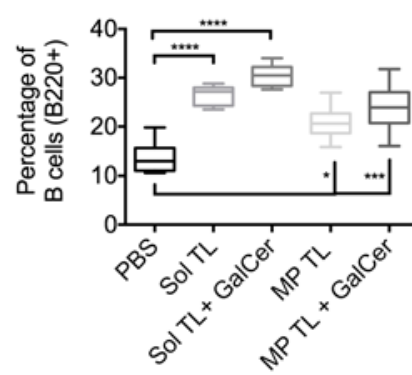

F

Anti lysate-IgG1 at day 128

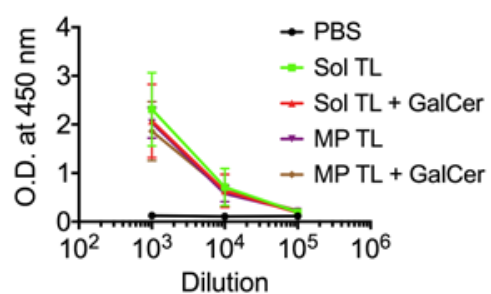

D Anti lysate-lgG1 at day 21

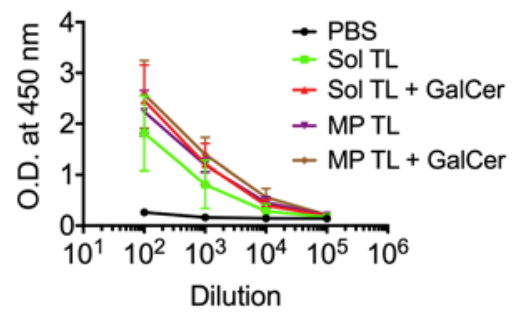

G Anti lysate-lgG2a at day 128

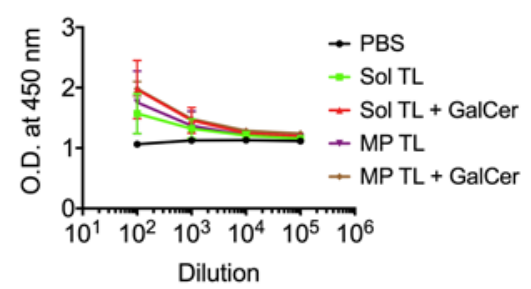

Figure 4. Humoral responses for various therapeutic A20 lysate vaccine formulations. (A) Confocal images of lymph node showing germinal center formation. Scale bar: $200 \mu \mathrm{m}$. (B) Box plot showing B220+GL7+ germinal center-forming B cells $(n=6)$. (C) Total B220 B cell percentage in lymph nodes ( $n$ = 6). (D) Anti-A20 tumor lysate IgG1 $(n=6)$ and (E) IgG2a $(n=4-6)$ antibody levels in serum at day 21 of the mechanistic studies (time line shown in Figure 3A). (F) Anti-A20 tumor lysate IgG1 ( $n=3-6 ;$ MP TL plus GalCer, $n=2)$ and (G) IgG2a antibody levels at day $128(n=3-6 ;$ MP TL plus GalCer, $n=2)$. Data represent mean $\pm \mathrm{SD}$. ${ }^{*} P<0.05,{ }^{* *} P<0.01,{ }^{* * *} P<0.001,{ }^{* * *} P<0.0001,1$-way ANOVA with Tukey multiple comparison tests.

antibody) are the best predictors for durable anti-A20 B cell lymphoma immune response for the Sol TL plus $\alpha$-GalCer group.

Therapeutic A20 lysate vaccines does not appear to cause autoimmunity. Autoimmune reactions are common adverse effects of various vaccines and immunotherapies. To assess any autoimmune reaction due to tumor lysate vaccines, we collected various organs at day 128 from mice of the therapeutic immunizations studies, as shown in Figure 2A, and performed histological studies. Histological analysis of tissue sections was done by a veterinary pathologist from Emory University, Atlanta, Georgia, USA. H\&E-stained tissue sections from various organs (liver, lung, heart, kidney, and colon; Supplemental Figure 9A) did not show any pathological changes or inflammatory reactions for any of the lysate vaccine groups, indicating absence of any autoimmune reaction. However, periarteriolar lymphoid sheaths of spleens from the lysate vaccine-treated mice showed hypercellularity, which could be due to germinal center response of A20 tumor vaccinated mice. Moreover, we measured anti-naive B cell lysate antibody levels in serum (day 128) to rule out generation of any self-reactive antibody. All the lysate vaccine groups showed very a low level of selfreactive antibody (Supplemental Figure 9, B and C).

\section{Discussion}

Research on therapeutic cancer vaccine has gained much interest following recent approval of the prostate vaccine sipuleucel-T (Provenge, Dendreon) by the FDA, and this is the first therapeutic vaccine ever 
A
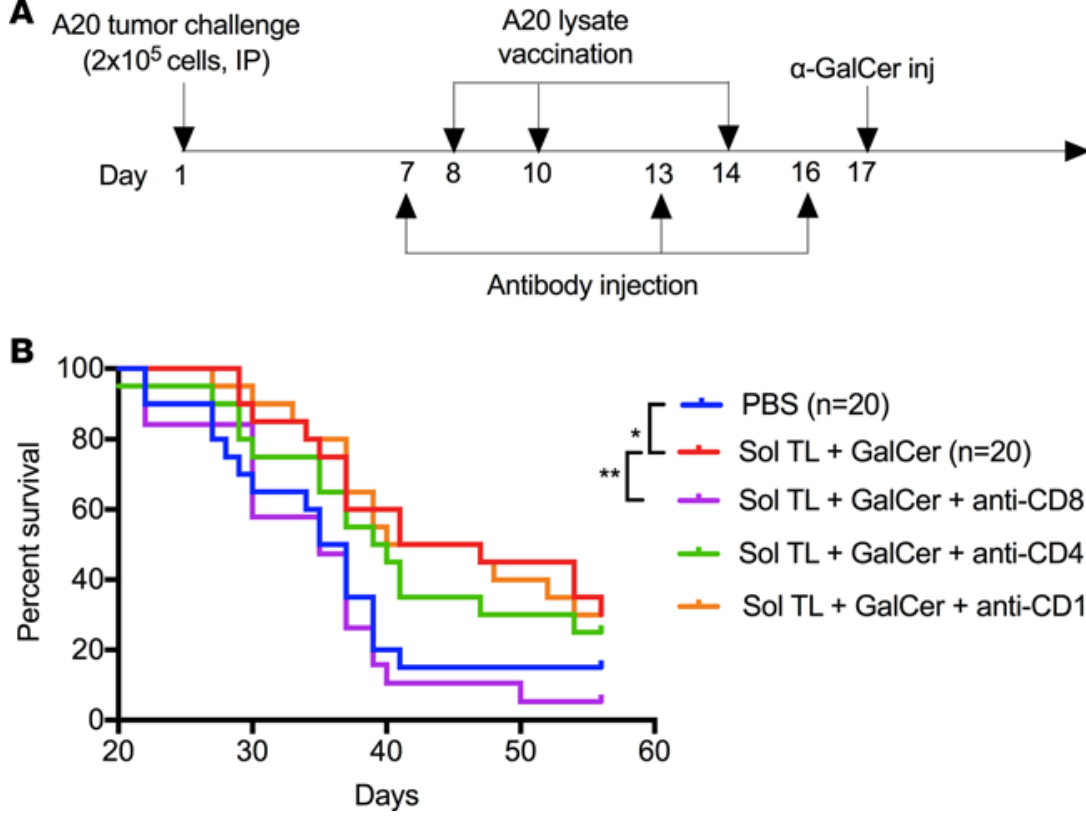

Figure 5. Therapeutic soluble tumor lysate plus $\alpha$-GalCer vaccine shows CD8 $T$ cell-dependent antitumor immunity in 7-day preestablished A20 B lymphoma model. (A) Time line showing therapeutic immunization and immune cell depletion/blocking antibody injection schedule. (B) Kaplan-Meier curve ( $n=19-20$ per group, pooled data from 2 independent experiments) showing survival of tumorchallenged mice for various antibodytreated (anti-mouse CD4, CD8, and CD1d antibodies) soluble tumor lysate plus $\alpha$-CalCer vaccine groups. ${ }^{*} P<0.05,{ }^{*} P<$ 0.01 , log-rank (Mantel-Cox) test.

approved by FDA (26). Historically, major efforts have been made to develop therapeutic vaccine that can enhance tumor antigen-specific immunity. However, this approach is only suitable for those few tumor types for which tumor antigens are known. Conversely, many tumors without known tumor antigens cannot be treated by tumor antigen-specific vaccine approach. So, concurrent approaches of using unidentified/undefined tumor-associated antigens (TAAs) in therapeutic vaccines have been explored $(27,28)$. Specifically, use of whole tumor cells or tumor lysate as a source of TAAs in therapeutic cancer vaccine has been found to be promising for various cancers $(28,29)$. The majority of TAA-based approaches have been reported for DC-based vaccines (20-22, 29), which are very expensive, time consuming, and process intensive. Recently, easy-to-make and inexpensive vaccines using irradiated whole tumor cells or tumor lysate combined with adjuvants have been reported to be promising for various cancers $(16,30,31)$. Here, we report a tumor lysate-based, directly injectable, personalized therapeutic vaccine for B cell lymphoma. Our goal was to develop an easy to prepare, highly effective, therapeutic vaccine for B cell lymphoma that can be easily translated to clinical use. We hypothesized that therapeutic vaccination with B lymphoma cell lysate combined with an appropriate adjuvant would enhance antitumor immune response against B lymphoma. We argued that the tumor lysate-based therapeutic vaccination approach for B lymphoma would be a viable alternative (less expensive, simple, and fast to prepare) to existing Id vaccines.

We used a syngeneic, murine, A20 B cell lymphoma model, which is known to be a poorly immunogenic murine model of human DLBCL and has been widely reported in literature $(10-14,32,33)$. We prepared A20 tumor lysate by a simple freeze-thaw method that took less than an hour. In an early treatment, low tumor burden, A20 B cell lymphoma model, soluble tumor lysate (Sol TL) only (without any adjuvant) showed impressive long-term immune protection against two lethal tumor challenges to $100 \%$ mice, indicating that the soluble A20 lysate vaccine triggers both effector and memory antitumor immune responses. On the other hand, polymeric particle surface-loaded A20 lysate with/without particulate-CpG adjuvant failed to provide immune protection; this could be due to the fact that not all the TAAs that are needed for immune protection were surface loaded on the particle. Next, we used a more aggressive, delayed-treatment A20 B lymphoma model, in which the initial lethal dose of A20 tumor cells was doubled (i.e., $2 \times 10^{5}$ cells instead of $1 \times 10^{5}$ cells used in our first study), and first immunization dose was delayed to day 8 . We also included various particulate TLR adjuvants (10) and a slow-release MP formulation of tumor lysate, previously shown to boost therapeutic effect of tumor lysate vaccine (31). Even though all the lysate vaccine formulations tested here prolonged median survival in the delayed-treatment model, TLR9/3-based particulate adjuvants failed to enhance therapeutic efficacy of lysate vaccine further. We have previously shown that MP-CpG-IL10 formulation skews Th1/Th2 cytokine balance in DCs and significantly enhances the protective immune response of Id DNA vaccine when combined with an injectable hydrogel-based 
A

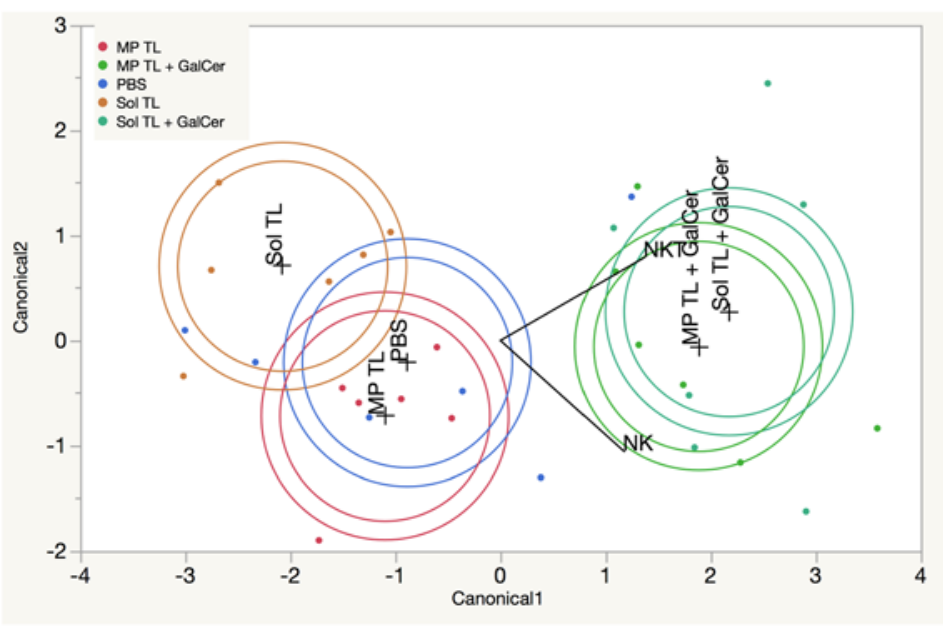

C

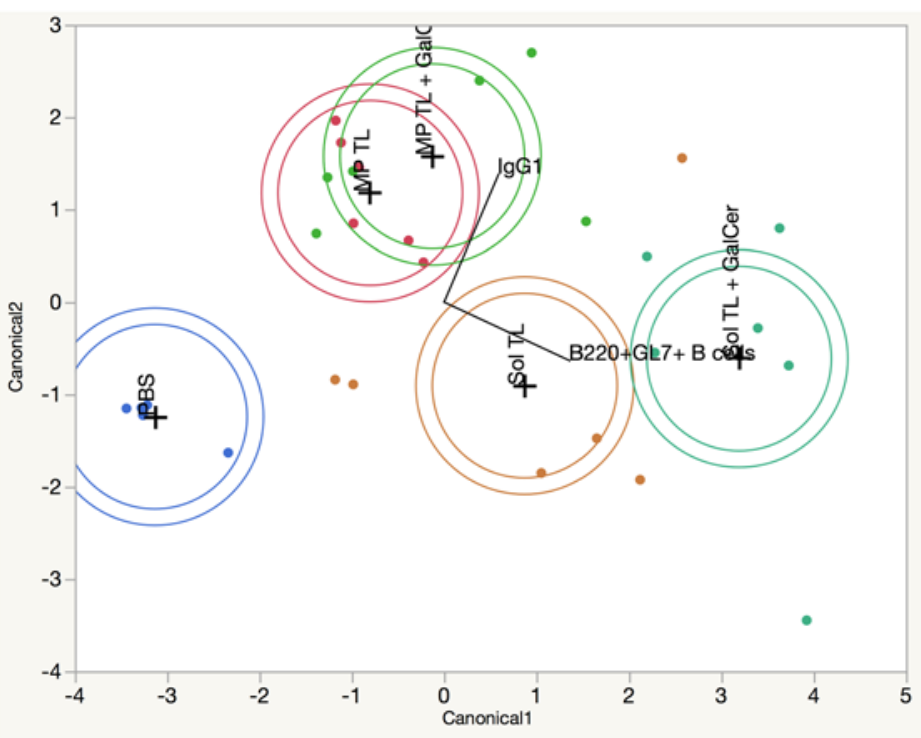

B Th1 and Th2 cells response

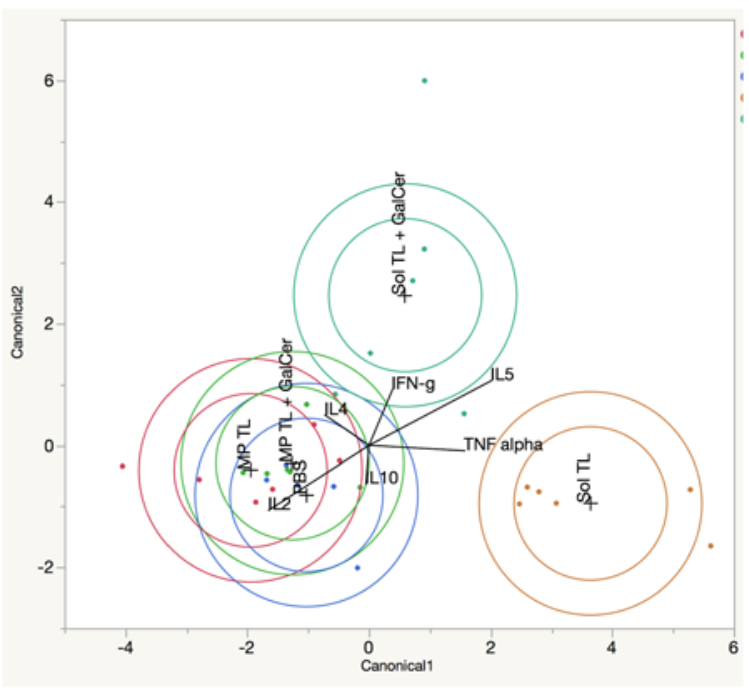

D

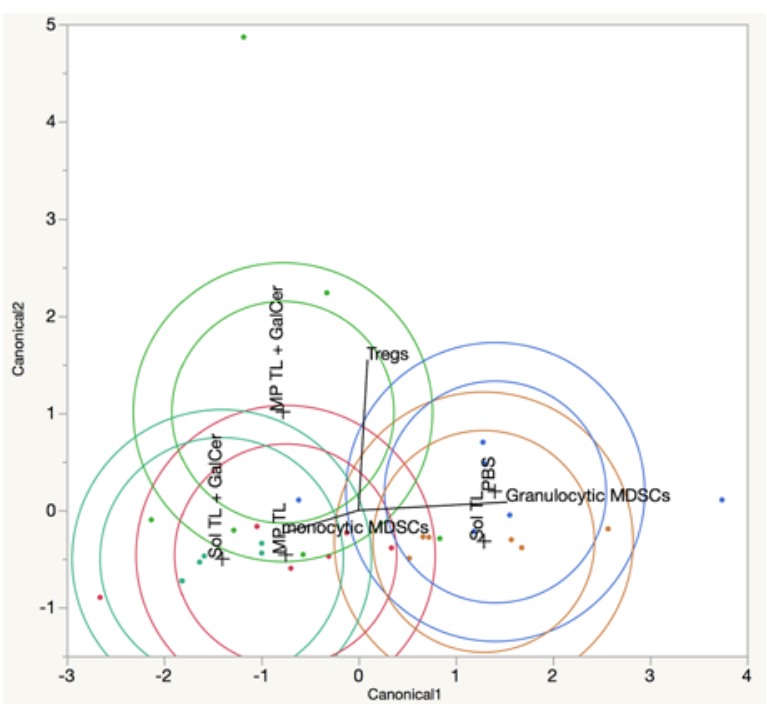

Figure 6. Linear discriminant analysis of immune responses for various therapeutic lysate vaccine groups. Canonical plots showing (A) NKT and NK cells responses, (B) Th1 and Th2 cytokine response, (C) humoral response, and (D) regulatory cell responses for various A20 tumor lysate vaccine groups.

synthetic immune priming center (sIPC) (10). In this study, we used MP-CpG-IL10 without sIPC to avoid complicated formulation processing, and this might lead to decreased antitumor immune response for MPCpG-IL10 formulations. However, the exact reasons why particulate CpG/poly (I:C)-based adjuvants did not help in increasing antitumor immunity for soluble A20 lysate vaccine need further investigations. Interestingly, the Sol TL vaccine group showed both CD8 T and NK cell responses, whereas MP TL predominantly had an NK cell response. These results prompted us to select an NKT cell agonist, $\alpha$-GalCer, which is known to indirectly enhance NK and T cell responses via NKT cell stimulation and was previously used as an adjuvant for autologous tumor cell vaccine for E $\mu$-myc and A20 B lymphoma $(24,25,34)$.

To evaluate long-term, durable immune protection effect of lysate vaccines, we used a delayed-treatment (7-day preestablished tumor) therapeutic immunization setting with tumor rechallenge in the mice that survived up to 56 days after first tumor challenge and long-term survival tracking until 128 days. Strikingly, $\alpha$-GalCer remarkably prolonged median survival when combined with Sol TL vaccine. Notably, slow-release formulation (MP TL) performed worse than Sol TL vaccine. Slow-release formulation was previously reported to enhance therapeutic efficacy of tumor lysate vaccines (31), but we did not see similar 
results for A20 lysate vaccine. Surprisingly, $\alpha$-GalCer treatment unexpectedly decreased the therapeutic efficacy of MP TL vaccines. However, it was clearly evident that the $\alpha$-GalCer plus Sol TL combination had durable, long-term immune protection against lethal A20 tumor challenges. Recently, Mattarollo et al. reported that irradiated autologous tumor cells loaded with $\alpha$-GalCer enhanced antitumor immunity against E $\mu$-myc lymphoma in the therapeutic immunization setting with 5- to 7-day preestablished tumors (25). However, a short-term antitumor immunity up to about 40 days was observed due to $\alpha$-GalCer-loaded therapeutic E $\mu$-myc tumor cell vaccine. In addition, Chung et al. had earlier shown that $\alpha$-GalCer-loaded irradiated A20 cell vaccine significantly enhanced survival of tumor-challenged mice compared with only irradiated A20 cell vaccine in therapeutic immunization settings (5- to 10-day preestablished tumor) (34). However, the irradiated cell vaccine (without $\alpha$-GalCer) failed to provide any immune protection against lethal tumor challenge, whereas our A20 lysate vaccine, even without $\alpha$-GalCer adjuvant, significantly enhanced survival of lethally challenged mice compared with the PBS control group, indicating superior immunogenicity of A20 lysate vaccine over irradiated A20 cell vaccine.

Further, our mechanistic studies show that multiple arms of immune systems were activated due to A20 tumor lysate vaccines, and the types of immune cells involved and degree of their stimulation/inhibition varied across different formulations/treatment regimes. Most importantly, the Sol TL plus $\alpha$-GalCer combination enhanced cellular (NKT and CTL) and humoral (germinal center and antitumor lysate IgG1 and IgG2a) responses and concurrently decreased immune-regulatory cells known to negatively influence antitumor immune responses; it seems that the cooperative interactions of all these immune responses lead to augmented antitumor immunity for the Sol TL plus $\alpha$-GalCer vaccine. Further, T cell depletion studies indicated that the tumor protective response of Sol TL plus $\alpha$-GalCer vaccine were CD8 T cell dependent. To identify critical immune biomarkers for long-term protection against A20 B cell lymphoma, we did multivariate analysis (LDA and PCA) $(35,36)$ of mechanistic and median survival data for various lysate vaccine groups. LDA and PCA identified that the germinal center B cell and Th1 (IFN- $\gamma+$ IgG2a) responses could be used as a predictive biomarker for long-term durable antitumor immune responses of Sol TL plus $\alpha$-GalCer vaccines against B cell lymphoma.

Overall, here we have shown that soluble tumor lysate combined with $\alpha$-GalCer adjuvant provides longterm durable immune protection against B cell lymphoma. Soluble A20 tumor lysate plus $\alpha$-GalCer vaccine activates multiple arms of innate and adaptive immune systems, such as cellular (CTL, NKT, IFN- $\gamma$, and IL4) and humoral immune response (germinal center and antitumor lysate IgG1 and IgG2a antibodies); shows CD8 T cell-dependent antitumor immunity; and does not provoke autoimmune reactivity. Germinal center response and Th1-type humoral response are the best predictive biomarkers for long-term, durable immune protection against B cell lymphoma. As the preparation of tumor lysate is a very simple (freezethaw method), quick (takes only about an hour), and does not need any expensive technology and expertise, soluble tumor lysate plus $\alpha$-GalCer vaccines would be easily translatable and more affordable clinically relevant therapeutic vaccines for B cell lymphoma compared with existing Id protein/DNA vaccines.

\section{Methods}

Study design. Here, our overarching goal was to develop a simple, clinically relevant therapeutic vaccine for B cell lymphoma that can provide durable, long-term immune protections against lethal tumor challenges. We prepared tumor lysate by a simple freeze-thaw method, used it as a source of TAAs, and tested its therapeutic efficacy with/without various molecular and particulate adjuvants in a poorly immunogenic, syngeneic, murine model of A20 B cell lymphoma that closely resembles human DLBCL. In our first experiment, we used a less aggressive therapeutic immunization setting (1-day preestablished A20 tumor model), in which mice (BALB/C, 6-10 mice per group) were first challenged with a lethal dose of A20 B lymphoma cells and then immunized with 3 doses of A20 lysate with/without adjuvants at days 2, 7, and 15. To see long-term immune protection effect of various lysate vaccine formulations, the mice were rechallenged with a double dose of A20 cells at day 80 and the survival of mice was recorded up to day 160. Next, we used an aggressive therapeutic immunization setting (7-day preestablished A20 tumor model), in which mice (BALB/C, 10 mice per group) were first challenge with a double dose of A20 B lymphoma cells compared with the first immunization study and then treated with 3 doses of various A20 lysate formulations at days 8, 10, and 14. In selective groups, in which an NKT cell agonist was used as an adjuvant, mice were injected (i.p.) with the adjuvant at day 17. Thereafter, at day 56, the surviving mice were rechallenged with the lethal dose of A20 cells, and the survival of mice was recorded till day 128. For mechanistic studies, 5-6 mice per group were sacrificed 
at day 21 of the therapeutic immunization studies, and lymph node, spleen, and serum was collected and analyzed for cellular and humoral immune responses. Long-term humoral responses of various lysate vaccine formulations were also analyzed using sera from the surviving mice at day 128 . Histopathological studies of various organs of mice at day 128 were done to evaluate for autoimmune reactions due to A20 lysate vaccinations. Neither therapeutic nor mechanistic mouse experiments were performed blind.

Preparation of A20 tumor lysate vaccine formulations. A20 cells (ATCC TIB-208) were purchased from ATCC and used for tumor challenge studies as well as lysate vaccine preparation. A20 tumor lysate was prepared by the freeze-thaw method (37). Briefly, A20 cells, cultured in complete growth media (RPMI $1640+10 \%$ FBS $+1 \%$ penicillin-streptomycin) under standard cell culture conditions, were collected by centrifugation, washed twice with PBS, and finally resuspended at $2 \times 10^{8}$ to $4 \times 10^{8}$ cells $/ \mathrm{ml}$ in the PBS. The cells were then subjected to 4 freeze-thaw cycles (freezing in liquid nitrogen and thawing at $37^{\circ} \mathrm{C}$ in a water bath), and the lysate was centrifuged at $12,000 \mathrm{gx}$ for 20 minutes at $4^{\circ} \mathrm{C}$. Finally, supernatant was collected and used as soluble tumor lysate (Sol TL) vaccine. Doses of the soluble tumor lysate were expressed as protein content of the lysate. The protein concentration of A20 tumor lysate was measured using Micro BCA assay (Thermo Scientific) using BSA as standard.

A20 tumor lysate encapsulated polymer MP(MP TL) was prepared using water-oil-water emulsion, solvent evaporation method. Briefly, a biodegradable polymer, poly (lactic-coglycolic acid) (PLGA, RG $502 \mathrm{H}$ resomer, Evonik), was used for MP TL preparation. Briefly, $200 \mathrm{mg}$ PLGA (acid end-capped) was first dissolved in $7 \mathrm{ml}$ methylene chloride, $300 \mu \mathrm{l} \mathrm{A20}$ lysate solution with $10 \mathrm{mg}$ lysate protein was added to the PLGA solution, and the mixture was homogenized at 10,000 rpm for 2 minutes using a PRO Scientific D series benchtop homogenizer. Then, the primary emulsion was added into $50 \mathrm{ml}$ of $1 \%$ poly vinyl alcohol solution and homogenized for 2 minutes at 10,000 rpm, followed by solvent evaporation for 4 hours. The MPs were then collected by centrifugation at 3,000 $\mathrm{g}$ for 20 minutes and washed twice with deionized water, lyophilized, and stored at $-20^{\circ} \mathrm{C}$. The MP TL formulations were characterized for size and zeta potential using a Zeta-sizer (Malvern). Amounts of encapsulated A20 lysate protein in MP TL were measured by Micro BCA assay following digestion of $5 \mathrm{mg}$ particles in $1 \mathrm{ml}$ of solution containing $100 \mathrm{mM} \mathrm{NaOH}$ and $0.05 \%$ sodium dodecyl sulphate (37). For MP TL-CpG-IL10 siRNA formulation, the surface of MP TL was further modified with a polycation, PEI (branched, MW 70,000, Polysciences), using EDC/NHS chemistry and then surface loaded with a TLR9 agonist, CpG, and IL10 siRNA by electrostatic interaction as we reported previously (10). In addition, we have used our previously reported various particulate adjuvants [PLGA-PEI MP surface loaded with CpG and IL10 siRNA, and CpG and poly $(\mathrm{I}: \mathrm{C})]$ (10) with soluble tumor lysates to enhance antitumor immune response of Sol TL vaccines. We also prepared surface-loaded tumor lysate (TL-MP) or dual tumor lysate plus CpG (TL-CpG-MP) MP formulations by electrostatic interactions between soluble tumor lysate/CpG and cationic PLGA-PEI particles. In selective vaccination groups, we used an NKT cell agonist, $\alpha$-GalCer (Avanti Polar Lipids) along with Sol TL and MP TL formulations to further boost their antitumor immune response.

Therapeutic immunization studies. Therapeutic efficacy of various A20 tumor lysate vaccine formulations was evaluated in two different therapeutic immunization settings, i.e., in 1-day preestablished and 7-day preestablished A20 syngeneic tumor models in BALB/C mice. In our first experiment, we used a 1-day preestablished A20 tumor model to test the therapeutic efficacy of soluble A20 tumor lysate with/without molecular adjuvant (e.g., soluble CpG) or particulate adjuvants (TL-MP, TL-CpG-MP, or TL-MP plus CpG-MP) as compared with the PBS-injected control group. In this experiment, BALB/C mice $(n=6-10)$ were first challenged with a lethal dose of A20 cells $\left(1 \times 10^{5}\right.$ A20 cells, i.p. $)$ and then immunized with various tumor lysate formulations (dose, $100 \mu \mathrm{g}$ A20 lysate protein, $50 \mu \mathrm{g} \mathrm{CpG} \mathrm{s.c.)} \mathrm{at} \mathrm{days} \mathrm{2,} \mathrm{7,} \mathrm{and} \mathrm{15.} \mathrm{Sur-}$ vival of mice was tracked thereafter. At day 80, a second tumor challenge with a double dose of A20 tumor cells $\left(2 \times 10^{5}\right.$ A20 cells, i.p.) was performed and the survival of mice was tracked up to 160 days. Further therapeutic studies were done in a highly aggressive, 7-day preestablished A20 B lymphoma model. Briefly, BALB/C mice $(n=10)$ were first challenged with a lethal dose of A20 tumor cells $\left(2 \times 10^{5}\right.$ A20 cells, i.p.) and then immunized with various formulations s.c. at day 8, 10, and 14 with $100 \mu \mathrm{g}$ A20 lysate protein (Sol TL or MP TL) with/without various particulate adjuvants [dose, $50 \mu \mathrm{g} \mathrm{CpG,} 50 \mu \mathrm{g}$ IL10 siRNA, and $50 \mu \mathrm{g}$ poly (I:C)]. In $\alpha$-GalCer treatment groups, a single i.p. injection with $2 \mu \mathrm{g} \alpha$-GalCer was done at day 17. For some groups, a second lethal challenge with $2 \times 10^{5} \mathrm{~A} 20$ cells, i.p., at day 56 was done and survival was tracked up to 128 days. For all the therapeutic studies, Median survival and Kaplan-Meier survival curves were generated, and log-rank (Mantel-Cox) tests were performed to compare survival between various groups using GraphPad Prism 6 software. 
Mechanistic studies. Mechanistic studies were done in therapeutic immunization setting using a 7-day preestablished A20 B lymphoma model (as described above). In a typical experiment, BALB/C mice ( $n=$ 5-6) were first injected with $2 \times 10^{5}$ A20 tumor cells on day 1 and then immunized with the formulations on day 8,10 , and 14 (as described above). A single i.p. injection with $2 \mu \mathrm{g} \alpha$-GalCer was done at day 17 only in $\alpha$-GalCer treatment groups. On day 21 , all the mice were sacrificed and spleen, inguinal lymph nodes, and blood (for serum) were collected and analyzed further for cellular and humoral immune responses using flow cytometry and ELISA.

Flow cytometry. Splenocytes and lymph nodes cells were analyzed for various immune cell populations, $\mathrm{CD}^{+} \mathrm{CD}^{+} \mathrm{T}$ cells, $\mathrm{CD}^{+} \mathrm{CD}^{+} \mathrm{T}$ cells, germinal center-forming B cells $\left(\mathrm{B} 220^{+} \mathrm{GL} 7^{+}\right)$, MDSCs $\left(\mathrm{CD} 11 \mathrm{~b}^{+} \mathrm{Gr} .1^{+}\right)$, Tregs $\left(\mathrm{CD} 4^{+} \mathrm{CD} 25^{+} \mathrm{FoxP}^{+}\right)$, NK cells $\left(\mathrm{CD} 3^{-} \mathrm{CD} 49 \mathrm{~b}^{+}\right)$and NKT cells $\left(\mathrm{TCR} \beta^{+} \mathrm{CD} 1 \mathrm{~d}-\right.$ tetramer ${ }^{+}$) using a BD LSRII flow cytometer following staining with specific anti-mouse antibodies.

CTL assay. CTL assays were performed using a Granzyme B assay kit (GranToxiLux kit, OncoImmunin) as we reported previously (11). Briefly, effector $\mathrm{CD}^{+}$or $\mathrm{CD}^{+} \mathrm{T}$ cells magnetically isolated (negatively sorted using magnetic isolation kits [CD4+ T Cell Isolation Kit, mouse, and CD8a+ T Cell Isolation Kit, mouse] from Miltenyi Biotec) from pooled splenocytes of various mechanistic study groups were coincubated with target A20 tumor cells (targets) at effector-to-target ratios of 20:1 for 45 minutes. Following incubation with a fluorogenic substrate for granzyme B, A20 target cells were analyzed for intracellular granzyme B activity using a BD LSRII flow cytometer.

Splenocyte restimulation studies. Splenocytes $\left(1 \times 10^{6}\right.$ cells/well of 96-well plates $)$ from immunized mice were restimulated ex vivo with A20 tumor lysate (100 $\mu$ g lysate protein/well of 96-well plates) for 3 days and stained and analyzed for IFN- $\gamma^{+} / \mathrm{IL}^{+}, \mathrm{CD}^{+} \mathrm{CD}^{+}$, and $\mathrm{CD} 3^{+} \mathrm{CD} 4^{+} \mathrm{T}$ cells using a BD LSRII flow cytometer. Further, culture media were analyzed for various Th1/Th2 cytokines using a multiplex kit (BioPlex Mouse Cytokine Kit) and a Luminex MAGPIX instrument.

Measurement of antibody levels by ELISA. Serum antibody levels were measured by ELISA using plates precoated with A20 tumor lysate/naive B cell lysate $(100 \mu \mathrm{g} / \mathrm{ml})$ or A20 Id protein $(10 \mu \mathrm{g} / \mathrm{ml})$ or MuLV capsid p30 or glycosylated Gag polyprotein ( $5 \mu \mathrm{g} / \mathrm{ml}$; Bioclone Inc.). Optical density for various serum dilutions was measured at $450 \mathrm{~nm}$.

Antibody specificity to A20 tumor cells. A20 cells were incubated with diluted serum (1:100 in FACS buffer) for 30 minutes and then stained with anti-mouse fluorescent IgG1 and IgG2a antibody and analyzed using a BD Accuri flow cytometer.

Immune cell depletion studies. For T cell depletion, $200 \mu \mathrm{g}$ anti-mouse CD8 $\alpha$ (clone 2.43, Bioxcell) or anti-mouse CD4 (clone GK1.5, Biolegend) was injected i.p. at indicated time points, as shown in Figure 5A (38). Similarly, $200 \mu \mathrm{g}$ anti-mouse CD1d (clone $20 \mathrm{H} 2$, Bioxcell) was injected to block NKT cell activation by CD1d-presented $\alpha$-GalCer (39).

Immunostaining for germinal center. Lymph nodes were collected from mice of various mechanistic study groups at day 21 and embedded in Tissue-Tek OCT compound and flash frozen in liquid nitrogen. Then, thin sections were cut using a cryostat; stained for germinal center formation using anti-mouse antibodies for B220, GL7, and IgG1; and visualized using a confocal microscope.

Histological studies. At day 128 of therapeutic immunization studies, mice were sacrificed and various organs were collected and fixed in formalin, paraffin embedded, and stained with H\&E for histological analysis of any autoimmune reaction. Histological analysis was done by a veterinary pathologist at Emory University.

Statistics. Unpaired, 2-tailed $t$ test was used compare pairs of groups, and 1-way ANOVA with Tukey test was performed for comparison in multiple group experiments using GraphPad Prism 6 software. Logrank (Mantel-Cox) test was done to define significant difference in survival between two groups in the therapeutic studies. $P$ values of less than 0.05 were considered significant. All values in the graphs are reported as mean \pm SD. Multivariate analysis (e.g., LDA and PCA) of mechanistic and survival data was performed using JMP 12 software.

Study approval. All the in vivo experiments with mice were approved by the institutional animal care and use committees at the Georgia Institute of Technology and the University of Texas at Austin, Austin, Texas, USA.

\section{Author contributions}

PP, J. Leleux, and KR designed the research; PP, J. Leleux, and J. Liu performed the experiments; PP, J. Leleux, and KR analyzed and interpreted the data; and PP and KR wrote the manuscript. 


\section{Acknowledgments}

We are grateful to the Georgia Institute of Technology, The Center for ImmunoEngineering and the Georgia Research Alliance for funding this research. We are also thankful to the NIH-Tetramer facility at Emory University for kindly providing CD1d-tetramer. We thank Larry W. Kwak (MD Anderson Cancer Center, Houston, Texas, USA) for kindly providing A20 Id protein and his feedback.

Address correspondence to: Krishnendu Roy, Center for Immunoengineering at Georgia Tech, The Wallace H. Coulter Department of Biomedical Engineering at Georgia Tech and Emory, The Parker H. Petit Institute for Bioengineering \& Bioscience, IBB 2314, Georgia Institute of Technology, EBB 3018, 950 Atlantic Drive, Mail Code: 2000, Atlanta, Georgia 30332, USA. Phone: 404.385.6166. Email: krishnendu. roy@bme.gatech.edu.

1. Rizzieri DA, et al. Improved efficacy using rituximab and brief duration, high intensity chemotherapy with filgrastim support for Burkitt or aggressive lymphomas: cancer and Leukemia Group B study 10 002. Br J Haematol. 2014;165(1):102-111.

2. Kantarjian H, Thomas D, Wayne AS, O'Brien S. Monoclonal antibody-based therapies: a new dawn in the treatment of acute lymphoblastic leukemia. J Clin Oncol. 2012;30(31):3876-3883.

3. Baskar S, Kobrin CB, Kwak LW. Autologous lymphoma vaccines induce human T cell responses against multiple, unique epitopes. J Clin Invest. 2004;113(10):1498-1510.

4. Schuster SJ, et al. Vaccination with patient-specific tumor-derived antigen in first remission improves disease-free survival in follicular lymphoma. J Clin Oncol. 2011;29(20):2787-2794.

5. Caspar CB, Levy S, Levy R. Idiotype vaccines for non-Hodgkin's lymphoma induce polyclonal immune responses that cover mutated tumor idiotypes: comparison of different vaccine formulations. Blood. 1997;90(9):3699-3706.

6. Cohen S, Haimovich J, Hollander N. B-cell lymphoma and myeloma protection induced by idiotype vaccination with dendritic cells is mediated entirely by T cells in mice. J Immunother. 2005;28(5):461-466

7. Weng WK, Czerwinski D, Timmerman J, Hsu FJ, Levy R. Clinical outcome of lymphoma patients after idiotype vaccination is correlated with humoral immune response and immunoglobulin G Fc receptor genotype. J Clin Oncol. 2004;22(23):4717-4724.

8. Allegra A, et al. Vaccination strategies in lymphopro9liferative disorders: Failures and successes. Leuk Res. 2015;39(10):1006-1019.

9. Ruffini PA, Neelapu SS, Kwak LW, Biragyn A. Idiotypic vaccination for B-cell malignancies as a model for therapeutic cancer vaccines: from prototype protein to second generation vaccines. Haematologica. 2002;87(9):989-1001.

10. Pradhan P, et al. The effect of combined IL10 siRNA and CpG ODN as pathogen-mimicking microparticles on Th1/Th2 cytokine balance in dendritic cells and protective immunity against B cell lymphoma. Biomaterials. 2014;35(21):5491-5504.

11. Singh A, et al. An injectable synthetic immune-priming center mediates efficient T-cell class switching and T-helper 1 response against B cell lymphoma. J Control Release. 2011;155(2):184-192.

12. Donnou S, Galand C, Touitou V, Sautès-Fridman C, Fabry Z, Fisson S. Murine models of B-cell lymphomas: promising tools for designing cancer therapies. Adv Hematol. 2012;2012:701704.

13. Qin $\mathrm{H}$, et al. Vaccine site inflammation potentiates idiotype DNA vaccine-induced therapeutic T cell-, and not B cell-, dependent antilymphoma immunity. Blood. 2009;114(19):4142-4149.

14. Sakamaki I, et al. Lenalidomide enhances the protective effect of a therapeutic vaccine and reverses immune suppression in mice bearing established lymphomas. Leukemia. 2014;28(2):329-337.

15. Sondak VK, Sosman JA. Results of clinical trials with an allogenic melanoma tumor cell lysate vaccine: Melacine. Semin Cancer Biol. 2003;13(6):409-415.

16. Wu A, et al. In vivo vaccination with tumor cell lysate plus $\mathrm{CpG}$ oligodeoxynucleotides eradicates murine glioblastoma. J Immunother. 2007;30(8):789-797.

17. Yoshikawa T, et al. Vaccine efficacy of fusogenic liposomes containing tumor cell-lysate against murine B16BL6 melanoma. Biol Pharm Bull. 2006;29(1):100-104.

18. Ali OA, Huebsch N, Cao L, Dranoff G, Mooney DJ. Infection-mimicking materials to program dendritic cells in situ. Nat Mater. 2009;8(2):151-158.

19. Sondak VK, et al. Adjuvant immunotherapy of resected, intermediate-thickness, node-negative melanoma with an allogeneic tumor vaccine: overall results of a randomized trial of the Southwest Oncology Group. J Clin Oncol. 2002;20(8):2058-2066.

20. Maier T, et al. Vaccination of patients with cutaneous T-cell lymphoma using intranodal injection of autologous tumor-lysatepulsed dendritic cells. Blood. 2003;102(7):2338-2344.

21. Hira SK, Mondal I, Manna PP. Combined immunotherapy with whole tumor lysate-pulsed interleukin-15-activated dendritic cells and cucurbitacin I promotes strong CD8(+) T-cell responses and cures highly aggressive lymphoma. Cytotherapy. 2015;17(5):647-664.

22. Gatza E, Okada CY. Tumor cell lysate-pulsed dendritic cells are more effective than TCR Id protein vaccines for active immunotherapy of T cell lymphoma. J Immunol. 2002;169(9):5227-5235.

23. Kasturi SP, Sachaphibulkij K, Roy K. Covalent conjugation of polyethyleneimine on biodegradable microparticles for delivery of plasmid DNA vaccines. Biomaterials. 2005;26(32):6375-6385.

24. Gonzalez-Aseguinolaza G, et al. Natural killer T cell ligand alpha-galactosylceramide enhances protective immunity induced by malaria vaccines. J Exp Med. 2002;195(5):617-624.

25. Mattarollo SR, et al. NKT cell adjuvant-based tumor vaccine for treatment of myc oncogene-driven mouse B-cell lymphoma. Blood. 2012;120(15):3019-3029.

26. Cheever MA, Higano CS. PROVENGE (Sipuleucel-T) in prostate cancer: the first FDA-approved therapeutic cancer vaccine. Clin Cancer Res. 2011;17(11):3520-3526. 
27. Buonaguro L, Petrizzo A, Tornesello ML, Buonaguro FM. Translating tumor antigens into cancer vaccines. Clin Vaccine Immunol. 2011;18(1):23-34

28. Joshi VB, Geary SM, Gross BP, Wongrakpanich A, Norian LA, Salem AK. Tumor lysate-loaded biodegradable microparticles as cancer vaccines. Expert Rev Vaccines. 2014;13(1):9-15.

29. de Gruijl TD, van den Eertwegh AJ, Pinedo HM, Scheper RJ. Whole-cell cancer vaccination: from autologous to allogeneic tumor- and dendritic cell-based vaccines. Cancer Immunol Immunother. 2008;57(10):1569-1577.

30. Bencherif SA, et al. Injectable cryogel-based whole-cell cancer vaccines. Nat Commun. 2015;6:7556.

31. Gross BP, Wongrakpanich A, Francis MB, Salem AK, Norian LA. A therapeutic microparticle-based tumor lysate vaccine reduces spontaneous metastases in murine breast cancer. AAPS J. 2014;16(6):1194-1203.

32. Palmieri $\mathrm{C}$, et al. In vivo targeting and growth inhibition of the A20 murine B-cell lymphoma by an idiotype-specific peptide binder. Blood. 2010;116(2):226-238.

33. Song W, Levy R. Therapeutic vaccination against murine lymphoma by intratumoral injection of naive dendritic cells. Cancer Res. 2005;65(13):5958-5964.

34. Chung Y, Qin H, Kang CY, Kim S, Kwak LW, Dong C. An NKT-mediated autologous vaccine generates CD4 T-cell dependent potent antilymphoma immunity. Blood. 2007;110(6):2013-2019.

35. Roy S, et al. Multivariate statistical analyses demonstrate unique host immune responses to single and dual lentiviral infection. PLoS ONE. 2009;4(10):e7359.

36. Genser B, Cooper PJ, Yazdanbakhsh M, Barreto ML, Rodrigues LC. A guide to modern statistical analysis of immunological data. BMC Immunol. 2007;8:27.

37. Prasad S, et al. Polymer nanoparticles containing tumor lysates as antigen delivery vehicles for dendritic cell-based antitumor immunotherapy. Nanomedicine. 2011;7(1):1-10.

38. Andreasson K, Eriksson M, Tegerstedt K, Ramqvist T, Dalianis T. CD4+ and CD8+ T cells can act separately in tumour rejection after immunization with murine pneumotropic virus chimeric Her2/neu virus-like particles. PLoS ONE. 2010;5(7):e11580

39. Renukaradhya GJ, Khan MA, Vieira M, Du W, Gervay-Hague J, Brutkiewicz RR. Type I NKT cells protect (and type II NKT cells suppress) the host's innate antitumor immune response to a B-cell lymphoma. Blood. 2008;111(12):5637-5645 